\title{
Analysis of the Relationship Between Preoperative Arterial Oxygen Partial Pressure and Acute Kidney Injury after Surgery for Tetralogy of Fallot and Explore the Related Risk Factors
}

\section{Yaya Xu}

Xinhua Hospital Affiliated to the Medical School of Shanghai Jiaotong University

Xiaodong Zhu ( $\nabla$ xinhuaxiaodong@126.com )

Xinhua Hospital Affiliated to the Medical School of Shanghai Jiaotong University

Lili Xu

Xinhua Hospital Affiliated to the Medical School of Shanghai Jiaotong University

\section{Zhen Li}

Xinhua Hospital Affiliated to the Medical School of Shanghai Jiaotong University

\section{Lulu Cao}

Xinhua Hospital Affiliated to the Medical School of Shanghai Jiaotong University

\section{Wei Xie}

Xinhua Hospital Affiliated to the Medical School of Shanghai Jiaotong University

Yueniu Zhu

Xinhua Hospital Affiliated to the Medical School of Shanghai Jiaotong University

\section{Research article}

Keywords: hypoxic tolerance, acute kidney injury, cardiothoracic surgery, postoperative, pediatric

Posted Date: January 29th, 2021

DOI: https://doi.org/10.21203/rs.3.rs-154463/v1

License: (c) (i) This work is licensed under a Creative Commons Attribution 4.0 International License. Read Full License 


\section{Abstract}

Background: Acute kidney injury (AKI) is a severe complication of pediatric cardiothoracic surgery (CTS). It is debatable whether patients with the low preoperative arterial partial pressure of oxygen ( $\mathrm{PaO} 2)$ are more likely to develop AKI after surgery. The study aims to investigate the incidence and possible influencing factors of AKI in patients undergoning the radical operation of tetralogy of Fallot (TOF) with different preoperative oxygen partial pressure.

Methods: In this retrospective clinical study, 36 pediatric patients who underwent CTS were enrolled in this study. The patients were divided into 4 groups according to preoperative $\mathrm{PaO2}$. We examined the baseline data and outcomes of the study population among groups.

Results: Of the 36 patients, 17 developed AKI. Compared with the high preoperative PaO2 group, the low preoperative $\mathrm{PaO} 2$ group mostly had severe $\mathrm{AKI}$ and persistent, but there was no significant difference in AKI among groups (Pख0.05). In the 48-hour continuous monitoring after surgery, the oxygen metabolism indexes (PvaCO2/Ca-v02) were correlated with AKI and there were significant differences among the groups.

Conclusions: Low preoperative $\mathrm{PaO} 2$ does not significantly increase the incidence of $\mathrm{AKI}$ and $\mathrm{Pv}-\mathrm{aCO} 2 / \mathrm{Ca}-\mathrm{vO} 2$ is associated with postoperative AKI and persistent.

\section{Background}

Acute kidney injury (AKI) is a severe complication of pediatric cardiothoracic surgery (CTS). Previous literature has reported that the incidence of postoperative AKI is as high as $20-30 \%$ and it is associated with markedly increased mortality and adverse outcomes after CTS (1-4). In the long run, AKI after congenital heart disease (CHD) will not only affect the prognosis but also increase the overall medical burden, which is an urgent problem to be solved (5). Previous researches found that cyanotic heart disease (C-CHD) was the independent risk factor for the development of AKI after CTS (6-7). And a study indicated that Tetralogy of Fallot (TOF) was a severe and most common C-CHD for which the outcomes were more unfavourable than other types of simple congenital heart diseases (8). But a recent study showed that there was no difference in postoperative AKI incidence between patients with higher haematocrit (Hct) and those without (9). The view was expressed that C-CHD patients had adaptive changes due to chronic hypoxia and these changes might be beneficial to the protection of postoperative renal function (10). Evidence from basic studies, repetitive hypoxic preconditioning increases renal hypoxia-inducible factor 1-alpha mRNA and protein levels and decreases mitochondrial Bax translocation, cytochrome $\mathrm{c}$ release, and tubular apoptosis, which has supported that idea (11). This study aimed to explore the relationship between the degree of hypoxia before CTS and postoperative AKI and analyze the risk factors of postoperative AKI.

\section{Methods}

A flowchart of our study is included in Fig. 1. This retrospective, single-center study enrolled 46 patients with TOF aged between 1-month and 12-month, who underwent CTS from July 1, 2017, to September 30, 2019. The study was in strict accordance with the Declaration of Helsinki and International Ethical Guidelines for Health-related Research Involving Humans. Demographic and clinical data were retrieved from the patients' medical records. 
Inclusion criteria for patients were: (1) age from 1-month to 12-month; (2) TOF was confirmed by preoperative echocardiography and intraoperative findings. TOF consists of a tetrad, or a group of 4 defects, which are ventricular septal defect, pulmonary stenosis, overriding aorta, and right ventricular hypertrophy (12); (3) the first time to receive CTS treatment and undergo complete surgical repair. Complete surgical repair includes closing the ventricular septal defect, resecting muscle bundles within the right ventricular outflow tract with or without patch augmentation (13); (4) complete clinical information such as history and laboratory examination.

The exclusion criteria were: (1) preexisting renal dysfunction (RD) or requirement of renal replacement therapy before surgery. RD was defined as kidney injury denoted by pathological changes, or other indicators such as abnormal blood, urine, or imaging findings or an estimated glomerular filtration rate (eGFR) of less than $60 \mathrm{ml} / \mathrm{min} * 1.73 \mathrm{~m} 2$ for more than 3 months (14); (2) a history of nephrotoxic drug use within 7 days before surgery; (3)a lack of postoperative renal data; (4) delayed sternal closure or reoperation was required due to bleeding and other reasons.

Finally, 36 patients were included in the study, of which 17 had AKI. Diagnosis and staging of AKI were performed according to the Kidney Disease: Improving Global Outcomes (KDIGO) 2012 clinical practice guidelines (15). Patients scheduled for CTS should have preoperative tests including the complete history, transthoracic echocardiography, and blood test. Venous blood used for the preoperative blood test was collected via scalp vein needle from scalp vein or femoral vein. The radial artery catheter was needed to obtain a sample of arterial blood for gas analysis and invasive monitoring. A central venous catheter (CVC) was placed through the femoral venous or jugular vein and central venous pressure (CVP) was measured hourly.

All postoperative children were admitted to the pediatric intensive care unit (PICU) and had real-time monitoring, which included measurement of vital signs (temperature, blood pressure, pulse, and respiration rate), quantification of all fluid intake and output. Blood used for routine postoperative test were collected from indwelling arterial lines after CTS, and simultaneous blood samples were obtained from a central venous catheter and an arterial catheter at 48 hours after surgery. Echocardiographic reexamination was performed on the 7th day after the operation. Postoperative management, systematic monitor and programmatic therapy were performed based on Handbook of Pediatric Cardiac Surgical Intensive Care (16).

Baseline data including demographics, clinical manifestation, and laboratory data were extracted and analyzed. Preoperative data included weight, height, age, sex, serum creatinine (SCr), eGFR, arterial oxygen pressure (PaO2), Hct, hemoglobin ( $\mathrm{Hb})$, McGoon ratio, Nakata index, pulmonary arterial pressure (PAP), left ventricular ejection fraction (LVEF) and fractional shortening (LVFS); surgical characteristics were CTS time, cardiopulmonary bypass (CPB) time and American Society of Anesthesiologists grade (ASA). Postoperative data including temperature, heart rate (HR), mean arterial pressure (MAP), central venous pressure (CVP), lactic acid, arterial oxygen saturation ( $\mathrm{SaO} 2), \mathrm{PaO} 2$ and urine output were recorded up to the first and second 24 hours after admission to PICU. Simultaneous monitoring of venous blood gas at 48 hours after CTS. The highest vasoactive inotrope score (VIS) within postoperative 48 hours was used to reflect the application of vasoactive drugs after CTS (17). Lengths of PICU and hospital stay were also recorded. To better show the oxygen metabolism in tissues, we calculated central venous-to-arterial carbon dioxide difference (Pv-aCO2), central arterial-to-venous oxygen saturation difference (Sa-v02), central arterial-to-venous oxygen pressure difference (Pa-v02), arterial-tovenous oxygen content difference (Ca-v02), the rate of $\mathrm{Pv}-\mathrm{aCO} 2 / \mathrm{Ca}-\mathrm{vO} 2$, and oxygen extraction ratio (O2 ER) according to the following formulas (18-20): 


$$
\begin{aligned}
& \cdot \mathrm{CaO} 2=(1.34 \times \mathrm{SaO} 2 \times \mathrm{Hb})+(0.003 \times \mathrm{PaO} 2) \\
& \cdot \mathrm{CcvO} 2=(1.34 \times \mathrm{ScvO} 2 \times \mathrm{Hb})+(0.003 \times \mathrm{PcvO} 2) \\
& \cdot \mathrm{Ca}-\mathrm{vO} 2=\mathrm{CaO} 2-\mathrm{CcvO} 2 \\
& \cdot \mathrm{Pv}-\mathrm{aCO} 2=\mathrm{PcvCO} 2-\mathrm{PaCO} 2 \\
& \cdot \mathrm{Pv}-\mathrm{aCO} 2 / \mathrm{Ca}-\mathrm{vO} 2 \text { ratio }=\mathrm{Pv}-\mathrm{aCO} 2 / \mathrm{Ca}-\mathrm{vO} 2 \\
& \cdot \mathrm{Pa}-\mathrm{vO} 2=\mathrm{PaO} 2-\mathrm{PcvO} 2 \\
& \cdot \mathrm{Sa}-\mathrm{vO} 2=\mathrm{SaO} 2-\mathrm{ScvO} 2 \\
& \cdot \mathrm{O} 2 \mathrm{ER}=\mathrm{Ca}-\mathrm{vO} 2 / \mathrm{CaO} 2
\end{aligned}
$$

Shapiro-Wilk test was used to check the normality of the data; continuous variables with normal distribution were expressed in terms of mean and standard deviation. Group comparisons were analyzed by independent sample ttests or ANOVA test. The continuous variables with the non-normal distribution were expressed as median (P25 and P75) and were compared using non-parametric factorial Kruskal-Wallis sum-rank test. Frequencies and proportions were estimated for categorical variables and were compared using the chi-squared test or Fisher's test. Odds ratios (ORs) with 95\% confidence intervals (Cls) for the development of AKI in each group were calculated using a logistic regression model. Persistent AKI was defined as a continuance of AKI according to the KDIGO criteria beyond $48 \mathrm{~h}$ according to the consensus report of the ADQI 16 workgroup (21). Transient AKI was defined as $\mathrm{AKI}$ of less than $48 \mathrm{~h}$ duration. To investigate the relationship between preoperative $\mathrm{PaO} 2$ and postoperative AKI, Univariate analysis was performed on the general preoperative data, hemodynamic indexes and oxygen metabolism indexes of the 4 groups at 24 and 48 hours after operation and the incidence, severity, and duration of AKI in the 4 groups were compared. Subgroup analysis was performed to explore the relationship between oxygen metabolism index (Sa-v02, Pa-v02, Pv-aCO2, Ca-v02, Pv-aCO2/Ca-v02, O2ER) and persistent AKI. Single-factor regression analysis was performed to explore the risk factors with certain significance in univariate analysis $(\mathrm{P}<0.05)$ of postoperative $\mathrm{AKI}$ and persistent AKI. All statistical analyses were performed with SPSS version 23 (IBM Corp. Released (2015) IBM SPSS Statistics for Windows. IBM Corp., Armonk, NY). The difference was considered significant when the two-tailed P-value was less than 0.05 .

\section{Results}

Among the 36 patients, 21 were males and 15 were females. The median age was 6-month (range, 4-12 months). The median height was $67.0 \mathrm{~cm}$ (range, 55.0-80.0 cm) and the weight was $8.0 \mathrm{~kg}$ (range, 5.4-12.5 kg). Seventeen patients (47.2\%) developed AKI. For the present analyses, patients were categorized by preoperative PaO2 quartiles: Group 1, PaO2 of $>94.0 \mathrm{mmHg}(\mathrm{n}=9)$; Group 2, $\mathrm{PaO} 2$ of $>70.5$ to $\leq 94.0 \mathrm{mmHg}(\mathrm{n}=9)$; Group 3, $\mathrm{PaO} 2$ of $>70.5$ to $\leq 40.6 \mathrm{mmHg}(\mathrm{n}=9)$; Group $4, \mathrm{PaO} 2$ of $\leq 40.6 \mathrm{mmHg}(\mathrm{n}=9)$. The baseline values, clinical status, and surgical characteristics of all 36 patients were shown in Table 1. 
Table 1

Study population characteristics $(n=36)$

\begin{tabular}{|c|c|c|c|c|c|c|}
\hline \multirow[t]{4}{*}{ Characteristics } & \multicolumn{4}{|c|}{ Preoperative $\mathrm{PaO}_{2}(\mathrm{mmHg})$} & \multirow[t]{4}{*}{ Statistic } & \multirow{4}{*}{$\begin{array}{l}P \\
\text { value }\end{array}$} \\
\hline & Group 1 & Group 2 & Group 3 & Group 4 & & \\
\hline & $>94.0$ & $>70.5, \leq 94.0$ & $>40.6, \leq 70.5$ & $\leq 40.6$ & & \\
\hline & $(n=9)$ & $(n=9)$ & $(n=9)$ & $(n=9)$ & & \\
\hline \multicolumn{7}{|l|}{ Demographics } \\
\hline Gender (male,\%) & $7(19.4)$ & $5(13.9)$ & $3(8.3)$ & $6(16.7)$ & $\begin{array}{l}\chi^{2}= \\
3.842\end{array}$ & 0.343 \\
\hline Age (month) & $\begin{array}{l}10.0(6.0 \\
12.0)\end{array}$ & $6.0(6.0,7.0)$ & $6.0(5.0,6.0)^{a}$ & $\begin{array}{l}6.0(6.0 \\
8.0)\end{array}$ & $\begin{array}{l}H= \\
7.815\end{array}$ & 0.050 \\
\hline Height (cm) & $73.1 \pm 5.0$ & $66.5 \pm 3.0$ & $66.0 \pm 4.0$ & $66.1 \pm 6.2$ & $\begin{array}{l}F= \\
4.852\end{array}$ & 0.007 \\
\hline Weight (kg) & $\begin{array}{l}8.0(7.5 \\
9.3)\end{array}$ & $7.6(7.0,9.0)$ & $8.0(7.0,8.0)$ & $\begin{array}{l}8.0(7.0 \\
9.0)\end{array}$ & $\begin{array}{l}H= \\
1.501\end{array}$ & 0.682 \\
\hline $\begin{array}{l}\text { Body mass index } \\
\left(\mathrm{kg} / \mathrm{m}^{2}\right)\end{array}$ & $16.0 \pm 1.9$ & $18.7 \pm 2.7$ & $17.5 \pm 3.6$ & $18.2 \pm 4.8$ & $\begin{array}{l}F= \\
1.069\end{array}$ & 0.376 \\
\hline \multicolumn{7}{|l|}{ Preoperative testing } \\
\hline SCr(umol/L) & $23.8 \pm 3.7$ & $22.8 \pm 4.3$ & $23.2 \pm 2.8$ & $23.2 \pm 5.0$ & $\begin{array}{l}F= \\
0.093\end{array}$ & 0.963 \\
\hline $\operatorname{eGFR}\left(\mathrm{ml} / \mathrm{min} / 1.73 \mathrm{~m}^{3}\right)$ & $\begin{array}{l}101.4 \pm \\
13.2\end{array}$ & $98.5 \pm 23.2$ & $93.6 \pm 14.3$ & $96.3 \pm 22.7$ & $\begin{array}{l}F= \\
0.272\end{array}$ & 0.845 \\
\hline $\mathrm{PaO}_{2}(\mathrm{mmHg})$ & $\begin{array}{l}125.5 \pm \\
17.7\end{array}$ & $80.8 \pm 8.1$ & $59.6 \pm 9.1$ & $34.8 \pm 3.1$ & $\begin{array}{l}F= \\
112.982\end{array}$ & $<0.001$ \\
\hline $\mathrm{Hb}(\mathrm{g} / \mathrm{L})$ & $\begin{array}{l}115.0 \\
(98.0, \\
141.0)\end{array}$ & $\begin{array}{l}125.0(118.0, \\
132.0)\end{array}$ & $\begin{array}{l}\text { 144.0 (130.0 } \\
153.0)\end{array}$ & $\begin{array}{l}132.0 \\
(124.0, \\
144.0)\end{array}$ & $\begin{array}{l}H= \\
6.228\end{array}$ & 0.101 \\
\hline $\operatorname{Hct}(\%)$ & $\begin{array}{l}34.6(30.4, \\
41.20)\end{array}$ & $\begin{array}{l}36.7 \\
(34.5,41.3)\end{array}$ & $\begin{array}{l}42.8(37.8 \\
45.7)\end{array}$ & $\begin{array}{l}40.5(37.4, \\
34.0)\end{array}$ & $\begin{array}{l}H= \\
7.512\end{array}$ & 0.057 \\
\hline \multicolumn{7}{|l|}{$\begin{array}{l}\text { Preoperative } \\
\text { echocardiography }\end{array}$} \\
\hline LVEF (\%) & $68.1 \pm 7.1$ & $65.7 \pm 3.1$ & $68.4 \pm 6.8$ & $65.8 \pm 6.3$ & $\begin{array}{l}F= \\
0.539\end{array}$ & 0.659 \\
\hline LVFS (\%) & $36.1 \pm 6.7$ & $35.7 \pm 2.6$ & $36.2 \pm 5.3$ & $34.4 \pm 4.8$ & $\begin{array}{l}F= \\
0.232\end{array}$ & 0.873 \\
\hline
\end{tabular}

A two-tailed $P$ value of $<0.05$ was considered statistically significant;Compared with group $1,{ }^{\text {a }} P<0.05$

$\mathrm{PaO}_{2}=$ Arterial Partial Pressure of Oxygen; $\mathrm{CTS}=$ Cardiothoracic Surgery $; \mathrm{CPB}=$ Cardiopulmonary Bypass;

$\mathrm{SCr}=$ Serum Creatinine; eGFR = estimated Glomerular Filtration Rate; PICU = Pediatric Intensive Care Unit; Hct $=$ Hematocrit; $\mathrm{Hb}=$ Hemoglobin; $\mathrm{ASA}=$ American Society of Anesthesiologists; $\mathrm{PAP}=$ Pulmonary Artery

Pressure; LVEF = Left Ventricular Ejection Fraction; LVFS = Left Ventricular Fraction Shortening 


\begin{tabular}{|c|c|c|c|c|c|c|}
\hline \multirow[t]{4}{*}{ Characteristics } & \multicolumn{4}{|c|}{ Preoperative $\mathrm{PaO}_{2}(\mathrm{mmHg})$} & \multirow[t]{4}{*}{ Statistic } & \multirow{4}{*}{$\begin{array}{l}P \\
\text { value }\end{array}$} \\
\hline & Group 1 & Group 2 & Group 3 & Group 4 & & \\
\hline & $>94.0$ & $>70.5, \leq 94.0$ & $>40.6, \leq 70.5$ & $\leq 40.6$ & & \\
\hline & $(n=9)$ & $(n=9)$ & $(n=9)$ & $(n=9)$ & & \\
\hline PAP $(\mathrm{mmHg})$ & $71.2 \pm 21.9$ & $79.3 \pm 31.6$ & $79.4 \pm 26.9$ & $81.4 \pm 27.2$ & $\begin{array}{l}F= \\
0.250\end{array}$ & 0.860 \\
\hline $\begin{array}{l}\text { Nakata } \\
\text { index }\left(\mathrm{mm}^{2} / \mathrm{m}^{2}\right)\end{array}$ & $\begin{array}{l}128.3 \\
(127.3 \\
138.7)\end{array}$ & $\begin{array}{l}127.2(118.9 \\
138.6)\end{array}$ & $\begin{array}{l}122.8(114.5 \\
127.3)\end{array}$ & $\begin{array}{l}115.1 \\
(107.2 \\
123.2)\end{array}$ & $\begin{array}{l}H= \\
6.087\end{array}$ & 0.107 \\
\hline McGoon & $\begin{array}{l}1.5(1.3 \\
1.7)\end{array}$ & $1.4(1.3,1.7)$ & $1.3(1.3,1.4)$ & $\begin{array}{l}1.3(1.3 \\
1.3)\end{array}$ & $\begin{array}{l}H= \\
3.126\end{array}$ & 0.373 \\
\hline \multicolumn{7}{|l|}{$\begin{array}{l}\text { Surgical } \\
\text { characteristics }\end{array}$} \\
\hline CTS time (min) & $\begin{array}{l}119.0 \\
(109.0 \\
125.0)\end{array}$ & $\begin{array}{l}\text { 148.0 (130.0, } \\
163.0)\end{array}$ & $\begin{array}{l}164.0(135.0 \\
175.0)\end{array}$ & $\begin{array}{l}165.0 \\
(164.0 \\
220.0)^{a}\end{array}$ & $\begin{array}{l}H= \\
13.958\end{array}$ & 0.003 \\
\hline CPB time (min) & $\begin{array}{l}53.0(48.0 \\
58.0)\end{array}$ & $\begin{array}{l}65.0(60.0 \\
69.0)\end{array}$ & $\begin{array}{l}82.0(64.0 \\
88.0)\end{array}$ & $\begin{array}{l}86.0(77.0 \\
112.0)^{a}\end{array}$ & $\begin{array}{l}H= \\
12.740\end{array}$ & 0.005 \\
\hline \multicolumn{7}{|l|}{ ASA grade } \\
\hline$-\leq 2(\%)$ & $2(5.6)$ & 0 & $1(2.8)$ & $2(5.6)$ & \multirow{2}{*}{$\begin{array}{l}\chi^{2}= \\
2.603\end{array}$} & \multirow[t]{2}{*}{0.722} \\
\hline$-\geq 3(\%)$ & $7(19.4)$ & $9(25.0)$ & $8(22.2)$ & $7(19.4)$ & & \\
\hline \multicolumn{7}{|l|}{ Prognosis } \\
\hline PICU stay (days) & $\begin{array}{l}3.0(3.0 \\
4.0)\end{array}$ & $4.0(2.0,5.0)$ & $\begin{array}{l}5.0(4.0,7.0) \\
a\end{array}$ & $\begin{array}{l}4.0(3.0 \\
5.0)\end{array}$ & $\begin{array}{l}H= \\
9.529\end{array}$ & 0.023 \\
\hline Hospital stay (days) & $\begin{array}{l}14.0(11.0 \\
16.0)\end{array}$ & $\begin{array}{l}13.0(11.0 \\
15.0)\end{array}$ & $\begin{array}{l}16.0(15.0 \\
21.0)\end{array}$ & $\begin{array}{l}16.0(13.0 \\
18.0)\end{array}$ & $\begin{array}{l}H= \\
4.549\end{array}$ & 0.208 \\
\hline $\begin{array}{l}\text { Total hospitalization } \\
\text { expenses (yuan) }\end{array}$ & $\begin{array}{l}55113.3 \\
(53474.3, \\
60633.3)\end{array}$ & $\begin{array}{l}68372.6 \\
(61148.9 \\
76046.8)^{a}\end{array}$ & $\begin{array}{l}74039.3 \\
(71326.8 \\
78707.4)^{a}\end{array}$ & $\begin{array}{l}70783.0 \\
(66522.5 \\
74624.0)^{a}\end{array}$ & $\begin{array}{l}H= \\
17.543\end{array}$ & 0.001 \\
\hline \multicolumn{7}{|c|}{ A two-tailed $P$ value of $<0.05$ was considered statistically significant;Compared with group $1,{ }^{\text {a }} P<0.05$} \\
\hline \multicolumn{7}{|c|}{$\begin{array}{l}\mathrm{PaO}_{2}=\text { Arterial Partial Pressure of Oxygen; CTS = Cardiothoracic Surgery; CPB = Cardiopulmonary Bypass; } \\
\mathrm{SCr}=\text { Serum Creatinine; eGFR = estimated Glomerular Filtration Rate; PICU = Pediatric Intensive Care Unit; Hct } \\
\text { = Hematocrit; Hb = Hemoglobin; ASA = American Society of Anesthesiologists; PAP = Pulmonary Artery } \\
\text { Pressure; LVEF = Left Ventricular Ejection Fraction; LVFS = Left Ventricular Fraction Shortening }\end{array}$} \\
\hline
\end{tabular}


Table 2

Comparison of hemodynamic index and oxygen metabolism index among groups $(n=36)$

\begin{tabular}{|c|c|c|c|c|c|c|}
\hline \multirow[t]{4}{*}{ Characteristics } & \multicolumn{4}{|c|}{ Preoperative $\mathrm{PaO}_{2}(\mathrm{mmHg})$} & \multirow[t]{4}{*}{ Statistic } & \multirow{4}{*}{$\begin{array}{l}P \\
\text { value }\end{array}$} \\
\hline & Group 1 & Group 2 & Group 3 & Group 4 & & \\
\hline & & $>70.5, \leq 94.0$ & $>40.6, \leq 70.5$ & $\leq 40.6$ & & \\
\hline & $(n=9)$ & $(n=9)$ & $(n=9)$ & $(n=9)$ & & \\
\hline \multicolumn{7}{|l|}{ Temperature $\left({ }^{\circ} \mathrm{C}\right)$} \\
\hline Admission to PICU & $\begin{array}{l}36.5(36.0 \\
36.8)\end{array}$ & $\begin{array}{l}36.3(36.0, \\
36.7)\end{array}$ & $\begin{array}{l}36.4(36.2 \\
36.8)\end{array}$ & $\begin{array}{l}36.0(36.0 \\
36.4)\end{array}$ & $\begin{array}{l}H= \\
3.583\end{array}$ & 0.310 \\
\hline Postoperative $24 \mathrm{~h}$ & $37.5 \pm 0.5$ & $37.5 \pm 0.4$ & $37.0 \pm 0.7$ & $37.2 \pm 0.5$ & $\begin{array}{l}F= \\
0.167\end{array}$ & 0.686 \\
\hline Postoperative $48 \mathrm{~h}$ & $37.6 \pm 0.8$ & $37.0 \pm 0.7$ & $37.3 \pm 0.8$ & $37.1 \pm 0.6$ & $\begin{array}{l}F= \\
0.019\end{array}$ & 0.892 \\
\hline \multicolumn{7}{|l|}{ Heart rate(beats/min) } \\
\hline Admission to PICU & $\begin{array}{l}148.6 \pm \\
21.1\end{array}$ & $157.7 \pm 15.4$ & $163.9 \pm 11.9$ & $\begin{array}{l}154.3 \pm \\
15.3\end{array}$ & $\begin{array}{l}F= \\
3.049\end{array}$ & 0.090 \\
\hline Postoperative $24 \mathrm{~h}$ & $\begin{array}{l}130.6 \pm \\
15.0\end{array}$ & $151.0 \pm 13.1$ & $148.1 \pm 8.7$ & $145.9 \pm 9.4$ & $\begin{array}{l}F= \\
1.016\end{array}$ & 0.321 \\
\hline Postoperative $48 \mathrm{~h}$ & $\begin{array}{l}133.9 \pm \\
18.5\end{array}$ & $154.1 \pm 22.2$ & $158.8 \pm 14.6$ & $\begin{array}{l}148.3 \pm \\
14.7\end{array}$ & $\begin{array}{l}F= \\
1.320\end{array}$ & 0.259 \\
\hline \multicolumn{7}{|l|}{$M A P(m m H g)$} \\
\hline Admission to PICU & $67.4 \pm 8.0$ & $59.6 \pm 8.9$ & $66.3 \pm 7.3$ & $63.4 \pm 6.6$ & $\begin{array}{l}F= \\
0.457\end{array}$ & 0.504 \\
\hline Postoperative $24 \mathrm{~h}$ & $69.9 \pm 9.0$ & $60.6 \pm 6.8$ & $65.3 \pm 8.7$ & $66.4 \pm 8.9$ & $\begin{array}{l}F= \\
1.378\end{array}$ & 0.249 \\
\hline Postoperative $48 \mathrm{~h}$ & $\begin{array}{l}62.7(60.3 \\
74.0)\end{array}$ & $\begin{array}{l}60.3(57.3, \\
63.7)\end{array}$ & $\begin{array}{l}61.7(53.3 \\
62.0)\end{array}$ & $\begin{array}{l}66.7(60.3 \\
69.0)\end{array}$ & $\begin{array}{l}H= \\
4.615\end{array}$ & 0.202 \\
\hline \multicolumn{7}{|l|}{$C V P(m m H g)$} \\
\hline Admission to PICU & $\begin{array}{l}11.0(8.0 \\
11.0)\end{array}$ & $\begin{array}{l}9.0(9.0 \\
10.0)\end{array}$ & $\begin{array}{l}10.0(8.0 \\
11.0)\end{array}$ & $\begin{array}{l}10.0 \\
(8.0,10.0)\end{array}$ & $\begin{array}{l}H= \\
1.067\end{array}$ & 0.785 \\
\hline Postoperative $24 \mathrm{~h}$ & $9.3 \pm 1.4$ & $8.4 \pm 1.6$ & $10.6 \pm 1.9$ & $9.9 \pm 1.9$ & $\begin{array}{l}F= \\
1.315\end{array}$ & 0.287 \\
\hline
\end{tabular}

A two-tailed $\mathrm{P}$ value of $<0.05$ was considered statistically significant;Compared with group $1,{ }^{\text {a }} P<0.05$;

Compared with group $2,{ }^{\mathrm{b}} P<0.05$

$\mathrm{PICU}=$ Pediatric Intensive Care Unit; $\mathrm{PaO}_{2}=$ Arterial Partial Pressure of Oxygen; MAP = Mean Arterial Pressure; $\mathrm{CVP}=$ Central Venous Pressure; $\mathrm{SaO}_{2}=$ Arterial Oxygen Saturation; $\mathrm{P}_{\mathrm{v}-\mathrm{a}} \mathrm{CO}_{2} / \mathrm{C}_{\mathrm{a}-\mathrm{v}} \mathrm{O}_{2}=$ the ratio of central venous-to-arterial carbon dioxide difference $\left(\mathrm{Pv}_{\mathrm{aCO}}\right)$ to arterial-to-central venous $\mathrm{O}_{2}$ content difference (Ca$\left.\mathrm{vO}_{2}\right) ; \mathrm{O}_{2} \mathrm{ER}=$ Oxygen Extraction Ratio; $\mathrm{PAP}=$ Pulmonary Artery Pressure; LVEF = Left Ventricular Ejection Fraction; LVFS = Left Ventricular Fraction Shortening; VIS = Vasoactive Inotrope Score 


\begin{tabular}{|c|c|c|c|c|c|c|}
\hline \multirow[t]{4}{*}{ Characteristics } & \multicolumn{4}{|c|}{ Preoperative $\mathrm{PaO}_{2}(\mathrm{mmHg})$} & \multirow[t]{4}{*}{ Statistic } & \multirow{4}{*}{$\begin{array}{l}P \\
\text { value }\end{array}$} \\
\hline & Group 1 & Group 2 & Group 3 & Group 4 & & \\
\hline & $>94.0$ & $>70.5, \leq 94.0$ & $>40.6, \leq 70.5$ & $\leq 40.6$ & & \\
\hline & $(n=9)$ & $(n=9)$ & $(n=9)$ & $(n=9)$ & & \\
\hline Postoperative $48 \mathrm{~h}$ & $9.1 \pm 1.7$ & $10.3 \pm 1.9$ & $9.9 \pm 2.0$ & $9.9 \pm 2.1$ & $\begin{array}{l}F= \\
0.333\end{array}$ & 0.568 \\
\hline \multicolumn{7}{|c|}{ Lactic acid (mmol/L) } \\
\hline Admission to PICU & $\begin{array}{l}1.3(0.7 \\
1.5)\end{array}$ & $\begin{array}{l}1.3(1.2 \\
1.7)\end{array}$ & $\begin{array}{l}1.9(1.1 \\
2.2)\end{array}$ & $\begin{array}{l}1.5(1.0 \\
1.7)\end{array}$ & $\begin{array}{l}H= \\
3.230\end{array}$ & 0.357 \\
\hline Postoperative $24 \mathrm{~h}$ & $\begin{array}{l}0.7(0.5 \\
0.9)\end{array}$ & $\begin{array}{l}0.9(0.7 \\
1.2)\end{array}$ & $\begin{array}{l}1.0(0.7 \\
1.2)\end{array}$ & $\begin{array}{l}0.9(0.8 \\
1.2)\end{array}$ & $\begin{array}{l}H= \\
3.236\end{array}$ & 0.357 \\
\hline Postoperative $48 \mathrm{~h}$ & $\begin{array}{l}0.6(0.6 \\
0.7)\end{array}$ & $\begin{array}{l}0.7(0.6 \\
0.8)\end{array}$ & $\begin{array}{l}0.8(0.6 \\
0.9)\end{array}$ & $\begin{array}{l}0.7(0.6 \\
0.8)\end{array}$ & $\begin{array}{l}H= \\
1.003\end{array}$ & 0.800 \\
\hline \multicolumn{7}{|l|}{$\mathrm{SaO}_{2}(\%)$} \\
\hline Admission to PICU & $\begin{array}{l}99.7(99.4 \\
99.9)\end{array}$ & $\begin{array}{l}99.6(99.4 \\
100.1)\end{array}$ & $\begin{array}{l}99.9(99.7 \\
100.0)\end{array}$ & $\begin{array}{l}99.7(99.4 \\
100.3)\end{array}$ & $\begin{array}{l}H= \\
0.783\end{array}$ & 0.854 \\
\hline Postoperative $24 \mathrm{~h}$ & $\begin{array}{l}99.9(99.6 \\
100.1)\end{array}$ & $\begin{array}{l}99.9(97.9 \\
100.0)\end{array}$ & $\begin{array}{l}98.5(96.8 \\
99.5)\end{array}$ & $\begin{array}{l}99.3(99.2 \\
99.9)\end{array}$ & $\begin{array}{l}H= \\
6.736\end{array}$ & 0.081 \\
\hline Postoperative $48 \mathrm{~h}$ & $\begin{array}{l}99.7(99.6 \\
100.0)\end{array}$ & $\begin{array}{l}99.9(99.5 \\
100.3)\end{array}$ & $\begin{array}{l}98.6(96.7 \\
100.0)\end{array}$ & $\begin{array}{l}99.0(98.1 \\
99.7)\end{array}$ & $\begin{array}{l}H= \\
5.790\end{array}$ & 0.122 \\
\hline \multicolumn{7}{|l|}{$\mathrm{PaO}_{2}(\mathrm{mmHg})$} \\
\hline Admission to PICU & $\begin{array}{l}186.3 \pm \\
30.9\end{array}$ & $177.4 \pm 55.3$ & $161.4 \pm 55.8$ & $\begin{array}{l}155.0 \pm \\
57.3\end{array}$ & $\begin{array}{l}H= \\
0.716\end{array}$ & 0.550 \\
\hline Postoperative $24 \mathrm{~h}$ & $\begin{array}{l}175.2 \pm \\
57.9\end{array}$ & $175.4 \pm 86.0$ & $129.9 \pm 60.1$ & $\begin{array}{l}127.3 \pm \\
66.0\end{array}$ & $\begin{array}{l}H= \\
1.402\end{array}$ & 0.260 \\
\hline Postoperative $48 \mathrm{~h}$ & $\begin{array}{l}169.0 \pm \\
26.5\end{array}$ & $157.7 \pm 45.5$ & $121.4 \pm 64.7$ & $94.1 \pm 83.9$ & $\begin{array}{l}H= \\
3.030\end{array}$ & 0.044 \\
\hline \multicolumn{7}{|c|}{$\begin{array}{l}\text { Oxygen metabolism } \\
\text { index(Postoperative 48h) }\end{array}$} \\
\hline $\mathrm{Sa}-\mathrm{vO}_{2}(\mathrm{mmHg})$ & $3.5 \pm 1.9$ & $5.9 \pm 2.2$ & $9.4 \pm 4.0$ & $19.1 \pm 9.0$ & $\begin{array}{l}F= \\
1.076\end{array}$ & 0.373 \\
\hline
\end{tabular}

A two-tailed $P$ value of $<0.05$ was considered statistically significant;Compared with group $1,{ }^{\text {a }} P<0.05$; Compared with group $2,{ }^{\mathrm{b}} P<0.05$

$\mathrm{PICU}=$ Pediatric Intensive Care Unit; $\mathrm{PaO}_{2}=$ Arterial Partial Pressure of Oxygen; MAP = Mean Arterial Pressure; $\mathrm{CVP}=$ Central Venous Pressure; $\mathrm{SaO}_{2}=$ Arterial Oxygen Saturation; $\mathrm{P}_{\mathrm{v}-\mathrm{a}} \mathrm{CO}_{2} / \mathrm{C}_{\mathrm{a}-\mathrm{v}} \mathrm{O}_{2}=$ the ratio of central venous-to-arterial carbon dioxide difference $\left(\mathrm{Pv}-\mathrm{aCO}_{2}\right)$ to arterial-to-central venous $\mathrm{O}_{2}$ content difference (Ca$\left.\mathrm{VO}_{2}\right) ; \mathrm{O}_{2} \mathrm{ER}=$ Oxygen Extraction Ratio; PAP = Pulmonary Artery Pressure; LVEF = Left Ventricular Ejection Fraction; LVFS = Left Ventricular Fraction Shortening; VIS = Vasoactive Inotrope Score 


\begin{tabular}{|c|c|c|c|c|c|c|}
\hline \multirow[t]{4}{*}{ Characteristics } & \multicolumn{4}{|c|}{ Preoperative $\mathrm{PaO}_{2}(\mathrm{mmHg})$} & \multirow[t]{4}{*}{ Statistic } & \multirow{4}{*}{$\begin{array}{l}P \\
\text { value }\end{array}$} \\
\hline & Group 1 & Group 2 & Group 3 & Group 4 & & \\
\hline & $>94.0$ & $>70.5, \leq 94.0$ & $>40.6, \leq 70.5$ & $\leq 40.6$ & & \\
\hline & $(n=9)$ & $(n=9)$ & $(n=9)$ & $(n=9)$ & & \\
\hline $\mathrm{Pa}-\mathrm{vO}_{2}(\mathrm{mmHg})$ & $\begin{array}{l}31.0(24.0 \\
61.0)\end{array}$ & $\begin{array}{l}41.9(35.4 \\
68.4)\end{array}$ & $\begin{array}{l}25.6(16.2 \\
41.7)\end{array}$ & $\begin{array}{l}69.7(16.4 \\
91.9)\end{array}$ & $\begin{array}{l}H= \\
3.141\end{array}$ & 0.370 \\
\hline $\mathrm{Pv}-\mathrm{aCO}{ }_{2}(\mathrm{mmHg})$ & $\begin{array}{l}0.6(0.1 \\
3.1)\end{array}$ & $\begin{array}{l}5.3(1.0 \\
7.1)\end{array}$ & $\begin{array}{l}11.8(9.0 \\
15.6) \mathrm{a}\end{array}$ & $\begin{array}{l}23.4(18.8 \\
28.1) \mathrm{ab}\end{array}$ & $\begin{array}{l}H= \\
25.681\end{array}$ & $<0.001$ \\
\hline $\mathrm{Ca}-\mathrm{vO}_{2}(\mathrm{~mL} / \mathrm{dL})$ & $\begin{array}{l}5.8(4.3 \\
6.4)\end{array}$ & $\begin{array}{l}9.2(6.2 \\
10.1)\end{array}$ & $\begin{array}{l}\text { 13.4 (11.8, } \\
16.8) \text { a }\end{array}$ & $\begin{array}{l}29.8(20.8 \\
34.0) \mathrm{ab}\end{array}$ & $\begin{array}{l}H= \\
20.353\end{array}$ & $<0.001$ \\
\hline $\mathrm{Pv}-\mathrm{aCO}_{2} / \mathrm{Ca}-\mathrm{vO}_{2}$ & $\begin{array}{l}0.2(0.05 \\
0.48)\end{array}$ & $\begin{array}{l}0.6(0.4 \\
0.7)\end{array}$ & $\begin{array}{l}0.7(0.6 \\
1.3)\end{array}$ & $\begin{array}{l}0.8(0.7 \\
0.9) \text { a }\end{array}$ & $\begin{array}{l}H= \\
12.566\end{array}$ & $<0.001$ \\
\hline $\mathrm{O}_{2} \mathrm{ER}$ & $\begin{array}{l}0.04(0.03 \\
0.04)\end{array}$ & $\begin{array}{l}0.06(0.05 \\
0.07)\end{array}$ & $\begin{array}{l}0.09(0.07 \\
0.12) a\end{array}$ & $\begin{array}{l}0.24(0.14 \\
0.26) a b\end{array}$ & $\begin{array}{l}H= \\
24.142\end{array}$ & $<0.001$ \\
\hline \multicolumn{7}{|l|}{ Echocardiography } \\
\hline \multicolumn{7}{|l|}{ (Postoperative 7th day) } \\
\hline LVEF (\%) & $67.9 \pm 5.9$ & $68.0 \pm 7.8$ & $68.2 \pm 7.2$ & $65.9 \pm 4.3$ & $\begin{array}{l}F= \\
0.255\end{array}$ & 0.857 \\
\hline LVFS (\%) & $36.9 \pm 4.7$ & $36.1 \pm 5.9$ & $37.1 \pm 6.2$ & $35.0 \pm 3.2$ & $\begin{array}{l}F= \\
0.312\end{array}$ & 0.817 \\
\hline PAP $(\mathrm{mmHg})$ & $\begin{array}{l}11.8(11.0, \\
19.7)\end{array}$ & $\begin{array}{l}19.0(7.2 \\
23.8)\end{array}$ & $\begin{array}{l}19.0(16.3 \\
20.3)\end{array}$ & $\begin{array}{l}13.0(7.8 \\
17.6)\end{array}$ & $\begin{array}{l}H= \\
1.707\end{array}$ & 0.635 \\
\hline Nakata index $\left(\mathrm{mm}^{2} / \mathrm{m}^{2}\right)$ & $\begin{array}{l}145.2 \\
(140.3, \\
157.3)\end{array}$ & $\begin{array}{l}135.3 \\
(135.0, \\
157.6)\end{array}$ & $\begin{array}{l}141.2 \\
(138.7 \\
147.1)\end{array}$ & $\begin{array}{l}139.3 \\
(123.1, \\
145.0)\end{array}$ & $\begin{array}{l}H= \\
1.331\end{array}$ & 0.722 \\
\hline McGoon & $\begin{array}{l}1.6(1.5, \\
1.9)\end{array}$ & $\begin{array}{l}1.6(1.5, \\
1.7)\end{array}$ & $\begin{array}{l}1.6(1.5, \\
1.6)\end{array}$ & $\begin{array}{l}1.4(1.4 \\
1.5)\end{array}$ & $\begin{array}{l}H= \\
1.372\end{array}$ & 0.712 \\
\hline $\begin{array}{l}\text { VIS (highest within } 48 \\
\text { hours after CTS) }\end{array}$ & $\begin{array}{l}15.0(14.0 \\
15.0)\end{array}$ & $\begin{array}{l}13.0(10.0 \\
15.0)\end{array}$ & $\begin{array}{l}16.0(15.0 \\
18.0)\end{array}$ & $\begin{array}{l}18.0(12.0 \\
20.0)\end{array}$ & $\begin{array}{l}H= \\
4.914\end{array}$ & 0.178 \\
\hline \multicolumn{7}{|c|}{$\begin{array}{l}\text { A two-tailed } P \text { value of }<0.05 \text { was considered statistically significant; Compared with group } 1,{ }^{\text {a }} P<0.05 \text {; } \\
\text { Compared with group } 2,{ }^{b} P<0.05\end{array}$} \\
\hline \multicolumn{7}{|c|}{ 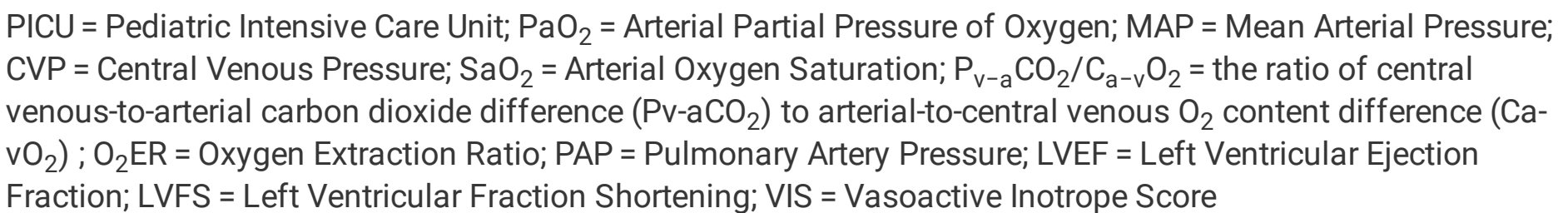 } \\
\hline
\end{tabular}

The oldest group is group 1 and there was a significant difference between group 1 and $3(P<0.05)$. Similarly, group 1 was the tallest among the groups. There was a significant difference in CTS time and CPB time between group 1 and 4; CTS time and CPB time were gradually increased $(P<0.05)$. The children in group 1 had average total hospitalization expenses of RMB 56140.6 (US\$8536.0) and length of stay in PICU of 2.9 days compared 
with the children in group 3 with expenses of RMB83770.8 (US\$12737.1) and PICU stays of 6.8 days $(P<0.05)$. The results showed that there were no significant differences in weight, sex, body mass index, preoperative SCr, preoperative eGFR, and preoperative echocardiography among groups (all $\mathrm{P}>0.05$ ).

The changes of hemodynamic indexes and oxygen metabolism indexes of the 4 groups at PICU, 24 hours and 48 hours after CTS were shown in Table 3. There were no statistically significant changes in the preoperative and 24, 48 hours postoperative records for temperature, HR, MAP, CVP lactic acid and SaO2 ( $P>0.05)$. $\mathrm{PaO} 2$ decreased gradually over time, but no significant differences were found between the values of preoperative and 24 hours after CTS among the groups. Patients in group 4 tended to have lower $\mathrm{PaO} 2$ at preoperative and 24,48 hours after CTS, and compared with group 1, patient in group 4 had higher $\mathrm{Pv}$-aCO2 (23.4 vs 0.6, $\mathrm{P}<0.05), \mathrm{Ca}-\mathrm{vO} 2$ (29.8 vs 5.8, $\mathrm{P}<0.05)$, Pv-aC02/Ca-v02 ratios (0.8 vs 0.2, $\mathrm{P}<0.05)$ and $02 \mathrm{ER}(0.24$ vs $0.04, \mathrm{P}<0.05)$ at 48 hours after CTS that met statistical significance (Table 3). There was no statistically significant difference in echocardiography (Postoperative 7th day) and VIS (highest within 48 hours after CTS).

Table 3

The occurrence and improvement of AKI in patients with congenital heart disease $(n=36)$

\begin{tabular}{|c|c|c|c|c|c|c|}
\hline \multirow[t]{4}{*}{ Characteristics } & \multicolumn{4}{|c|}{ Preoperative $\mathrm{PaO}_{2}(\mathrm{mmHg})$} & \multirow[t]{4}{*}{ Statistic } & \multirow[t]{4}{*}{$P$ value } \\
\hline & Group 1 & Group 2 & Group 3 & Group 4 & & \\
\hline & $>94.0$ & $>70.5, \leq 94.0$ & $>40.6, \leq 70.5$ & $\leq 40.6$ & & \\
\hline & $(n=9)$ & $(n=9)$ & $(n=9)$ & $(n=9)$ & & \\
\hline \multicolumn{7}{|l|}{$\mathrm{AKI}(\%)$} \\
\hline During hospitalization & $1(2.8)$ & $6(16.7)$ & $5(13.9)$ & $5(13.9)$ & $x^{2}=6.597$ & 0.079 \\
\hline Admission to PICU & $1(5.9)$ & $5(29.4)$ & $3(17.6)$ & $2(11.8)$ & $x^{2}=2.792$ & 0.475 \\
\hline Duration of $\mathrm{AKI} \geq 48 \mathrm{~h}$ & 0 & $2(11.8)$ & $4(23.5)$ & $4(23.5)$ & $\chi^{2}=4.460$ & 0.213 \\
\hline \multicolumn{7}{|l|}{ AKI severity (\%) } \\
\hline Stage 1 & $1(5.9)$ & $4(23.5)$ & $3(17.6)$ & $1(5.9)$ & \multirow[t]{3}{*}{$\chi^{2}=5.439$} & \multirow[t]{3}{*}{0.658} \\
\hline Stage 2 & 0 & $2(11.8)$ & $1(5.9)$ & $2(11.8)$ & & \\
\hline Stage 3 & 0 & 0 & $1(5.9)$ & $2(11.8)$ & & \\
\hline
\end{tabular}

The total incidence of acute kidney injury was $47.2 \%(n=36)$ and the incidence of AKI was significantly lower in group 1 ( $n=1,2.8 \%)$. Most patients developed postoperative AKI in the early period after CTS $(n=11,64 \%)$, and patients with low $\mathrm{PaO} 2$ (group 3 and 4 ) tended to have persistent $\mathrm{AKI}(\mathrm{n}=8,80 \%)$, however it was not statistically significant $(P>0.05)$.

Subgroup analysis was performed based on whether the patients were persistent or short AKI which was shown in Table 4. Patients with persistent AKI had significantly higher Pv-aCO2, Ca-v02, Pv-aCO2/Ca-vO2 and O2ER 
than those with short $\mathrm{AKI}(\mathrm{P}>0.05)$. Even after grouping by $\mathrm{PaO2}$, this phenomenon persisted and those values of group 3 and 4 were higher than group 1 and 2 .

Table 4

Comparison of oxygen metabolism index among groups $(n=17)$

\begin{tabular}{|c|c|c|c|c|c|c|c|c|}
\hline \multirow[t]{3}{*}{ Characteristics } & \multirow{3}{*}{$\begin{array}{l}\text { Short } \\
\text { AKI } \\
(n= \\
7)\end{array}$} & \multirow{3}{*}{$\begin{array}{l}\text { Persistent } \\
\text { AKI } \\
(n=10)\end{array}$} & \multirow[t]{3}{*}{ Statistic } & \multirow{3}{*}{$\begin{array}{l}P \\
\text { value }\end{array}$} & \multicolumn{2}{|c|}{ Group 1 and 2} & \multicolumn{2}{|c|}{ Group 3and 4} \\
\hline & & & & & $\begin{array}{l}\text { Short } \\
\text { AKI }\end{array}$ & $\begin{array}{l}\text { Persistent } \\
\text { AKI }\end{array}$ & $\begin{array}{l}\text { Short } \\
\text { AKI }\end{array}$ & $\begin{array}{l}\text { Persistent } \\
\text { AKI }\end{array}$ \\
\hline & & & & & $(n=5)$ & $(n=2)$ & $\begin{array}{l}(n= \\
2)\end{array}$ & $(n=8)$ \\
\hline \multicolumn{9}{|l|}{$\begin{array}{l}\text { Oxygen } \\
\text { metabolism } \\
\text { index }\end{array}$} \\
\hline \multicolumn{9}{|l|}{$\begin{array}{l}\text { (Postoperative } \\
48 h)\end{array}$} \\
\hline $\begin{array}{l}\mathrm{Sa}- \\
\mathrm{VO}_{2}(\mathrm{mmHg})\end{array}$ & $\begin{array}{l}5.6 \\
(4.8 \\
7.7)\end{array}$ & $\begin{array}{l}10.9(7.2 \\
15.2)\end{array}$ & $\begin{array}{l}H= \\
0.586\end{array}$ & 0.061 & $\begin{array}{l}5.0(4.8 \\
6.0)\end{array}$ & $\begin{array}{l}6.9(5.8, \\
7.9)\end{array}$ & $\begin{array}{l}6.7 \\
(5.6 \\
7.7)\end{array}$ & $\begin{array}{l}\text { 10.1 (7.7, } \\
15.2)\end{array}$ \\
\hline $\begin{array}{l}\mathrm{Pa}- \\
\mathrm{vO}_{2}(\mathrm{mmHg})\end{array}$ & $\begin{array}{l}41.2 \\
\pm \\
37.8\end{array}$ & $\begin{array}{l}50.2 \pm \\
31.7\end{array}$ & $\begin{array}{l}F= \\
0.107\end{array}$ & 0.600 & $\begin{array}{l}54.0 \pm \\
37.5\end{array}$ & $\begin{array}{l}39.8 \pm \\
40.5\end{array}$ & $\begin{array}{l}19.1 \\
\pm \\
10.0\end{array}$ & $\begin{array}{l}52.8 \pm \\
32.0\end{array}$ \\
\hline $\begin{array}{l}\mathrm{Pv}- \\
\mathrm{aCO}_{2}(\mathrm{mmHg})\end{array}$ & $\begin{array}{l}3.0 \\
(1.3, \\
12.0)\end{array}$ & $\begin{array}{l}\text { 12.6 } \\
23.1)\end{array}$ & $\begin{array}{l}H= \\
1.807\end{array}$ & 0.070 & $\begin{array}{l}2.2(1.3 \\
3.0)\end{array}$ & $\begin{array}{l}1.5(1.3 \\
1.6)\end{array}$ & $\begin{array}{l}13.4 \\
(12.0 \\
14.7)\end{array}$ & $\begin{array}{l}15.8 \\
(11.7 \\
23.2)\end{array}$ \\
\hline $\begin{array}{l}\text { Ca-vO }{ }_{2} \\
(\mathrm{~mL} / \mathrm{dL})\end{array}$ & $\begin{array}{l}8.2 \\
(7.7 \\
10.5)\end{array}$ & $\begin{array}{l}16.7 \\
(10.8 \\
51.3)\end{array}$ & $\begin{array}{l}H= \\
2.830\end{array}$ & 0.003 & $\begin{array}{l}8.2 \\
(7.7,8.3)\end{array}$ & $\begin{array}{l}10.0(8.7 \\
11.4)\end{array}$ & $\begin{array}{l}9.1 \\
(7.4 \\
10.5)\end{array}$ & $\begin{array}{l}33.3 \\
(13.3 \\
55.3)\end{array}$ \\
\hline $\begin{array}{l}\mathrm{Pv}-\mathrm{aCO}_{2} / \mathrm{Ca}- \\
\mathrm{vO}_{2}\end{array}$ & $\begin{array}{l}0.3 \\
(0.2 \\
0.7)\end{array}$ & $\begin{array}{l}0.4(0.3 \\
1.4)\end{array}$ & $\begin{array}{l}H= \\
2.537\end{array}$ & 0.040 & $\begin{array}{l}0.3(0.2 \\
0.4)\end{array}$ & $\begin{array}{l}0.3(0.2, \\
0.4)\end{array}$ & $\begin{array}{l}0.5 \\
(0.4 \\
0.9)\end{array}$ & $\begin{array}{l}1.5(1.4, \\
1.6)\end{array}$ \\
\hline $\mathrm{O}_{2} \mathrm{ER}$ & $\begin{array}{l}0.06 \\
(0.05 \\
0.08)\end{array}$ & $\begin{array}{l}0.11 \\
(0.07 \\
0.36)\end{array}$ & $\begin{array}{l}H= \\
2.205\end{array}$ & 0.025 & $\begin{array}{l}0.05 \\
(0.05 \\
0.06)\end{array}$ & $\begin{array}{l}0.07 \\
(0.06 \\
0.08)\end{array}$ & $\begin{array}{l}0.08 \\
(0.05 \\
0.08)\end{array}$ & $\begin{array}{l}0.24 \\
(0.08 \\
0.38)\end{array}$ \\
\hline \multicolumn{9}{|c|}{$\begin{array}{l}\text { A two-tailed } \mathrm{P} \text { value of }<0.05 \text { was considered statistically significant; } \mathrm{AKI}=\mathrm{Acute} \text { Kidney Injury; } \mathrm{PaO}_{2}= \\
\text { Arterial Partial Pressure of Oxygen; } \mathrm{SaO} \mathrm{O}_{2}=\text { Arterial Oxygen Saturation; } \mathrm{P}_{\mathrm{v}-\mathrm{a}} \mathrm{CO}_{2} / \mathrm{C}_{\mathrm{a}-\mathrm{v}} \mathrm{O}_{2}=\text { the ratio of central } \\
\text { venous-to-arterial carbon dioxide difference }(\mathrm{Pv}-\mathrm{aCO} \\
\left.\mathrm{vO}_{2}\right) ; \mathrm{O}_{2} \mathrm{ER}=\text { Oxygen Extraction Ratio }\end{array}$} \\
\hline
\end{tabular}

Predictors of AKI and persistent AKI in single-factor logistic regression were shown in Fig. 2 and Fig. 3, respectively. Single-factor logistic regression analysis revealed a significant correlation between the occurrence of an AKI with age, height, $\mathrm{Hb}$, Hct, PICU stay days, total hospitalization expenses, VIS, Pv-aCO2 and Pv-aCO2/Cav02, while only Pv-aCO2/Ca-v02 showed a significant correlation with persistent AKI. 


\section{Discussion}

$\mathrm{AKI}$ is one of the common and serious complications after CPB operation, which has a negative impact on the prognosis of patients and increases the fatality rate (21). Most studies believe that chronic hypoxia has a great effect on the organism, especially for the kidney and even put forward the concept of cyanotic nephropathy (CN) (22). Studies have indicated that CN occurs in about $30-50 \%$ of the patients with C-CHD. The incidence of AKI after CTS was as high as $47.2 \%$ in this study and 10 patients had persistent AKI according to the definition. Hence it is important to identify patients who are at risk of developing AKI after CTS. Preoperative symptoms, surgical interventions, and postoperative management are related to the postoperative AKI. Previous studies have clearly demonstrated that prolonged CTS time and CPB time were associated with the development of AKI (23$24)$, however, it was not the case in our study. We considered that this is because the advances in surgical techniques and perioperative management that the CTS time (range, 99 to 282 minutes) and CPB time (range, 40 to 127 minutes) were controlled within a relatively safe range and had led to dramatic improvements in survival outcomes for children with TOF. We also analyzed the hemodynamic indicators and oxygen metabolism indicators at the time of PICU admission, postoperative $24 \mathrm{~h}$ and $48 \mathrm{~h}$. The arterial blood and venous blood gas analysis were measured simultaneously after the 48 hours CTS, and oxygen transport data ( $\mathrm{Sa}-\mathrm{vO} 2, \mathrm{~Pa}-\mathrm{vO2}$, $\mathrm{Ca}-\mathrm{vO} 2, \mathrm{Pv}-\mathrm{aCO} 2, \mathrm{O} 2 \mathrm{ER}$ and Pv-aCO2 / Ca - v02) were calculated. Due to the lack of echocardiographic data in the study population at 48 hours after surgery, we were unable to directly calculate oxygen delivery and oxygen consumption, which was a major flaw of this study.

MAP, CVP and SO2 are commonly used as the indices of adequate tissue perfusion and oxygenation in patients, but tissue hypoxia may still exist even when these indices are within the normal range (25). And in this study, normalization of SO2 and PO2 does not exclude the persistence of tissue hypoperfusion and tissue hypoxia. Some studies have found out that Sa-vO2 is an excellent indicator of adequacy of cellular oxygenation, and it has shown a good correlation with oxygen delivery (26); $\mathrm{Ca}$ - vO2 can to some extent reflect poor tissue perfusion (27); In this study, Pv-aCO2 / Ca - v02 and Pv-aCO2 were independent risk factors for acute kidney injury, and $\mathrm{Pv}-\mathrm{aCO} 2 / \mathrm{Ca}$ - vO2 was associated with persistent AKI. These results are consistent with previous studies. Mekontso-Dessap A et al. found that the Pv-aCO2 / Ca - v02 ratio could detect global anaerobic metabolism in critically ill patients (28). As an index of the presence of anaerobic metabolism, its basic theory is: during anaerobic metabolism carbon dioxide production decreases less than oxygen consumption. The present study showed that a high $\mathrm{Pv}-\mathrm{aCO} 2 / \mathrm{Ca}-\mathrm{vO} 2$ ratio was associated with low survival rates at day $28(\mathrm{P}<0.005)$ (29). Mukai A et al. found that Pv-aCO2 and Pv-aCO2 / Ca-vO2 at the end of surgery had a superior ability for predicting postoperative complications (30)

The patients in this study were divided into 4 groups according to preoperative $\mathrm{PaO} 2$ and patients with low $\mathrm{PaO} 2$ had higher $\mathrm{Hb}$ and $\mathrm{Hct}$ due to chronic hypoxia. Multiple studies have shown that high $\mathrm{Hb}$ and Hct are associated with low cardiac output, increased blood viscosity, increased platelet aggregation, and impaired blood flow (31). Although there was no significant difference between the $\mathrm{Hb}$ and $\mathrm{Hct}$ among groups in this study, patients with higher $\mathrm{Hb}$ and $\mathrm{Hct}$ had longer CTS time and CPB times, longer postoperative PICU stays, higher VIS scores, and higher expenditures. However, there was no significant difference in the incidence of postoperative AKI among the 4 groups, several hypotheses might be used to justify this observed discrepancy between lung tissue and systemic responses: (1) following CTS, all patients were admitted to the PICU and received routine mechanical ventilation, vasoactive drugs, moderate rehydration, therefore, the mature surgical technique and rational postoperative management strategies were closely associated with the decreased of the incidence of AKI. (2) the 
short follow-up time and the small number of patients involved, it was necessary to extend the follow-up time and increase the number of patients included. Overall, patients in group 1 had less kidney damage among the groups, and patients in group 3 and 4 were more likely to have severe AKI and persistent AKI.

During chronic hypoxia, increases in red blood cell number or formation of new vasculature occur, adapting an organism to decreased oxygen conditions. In this study, we found that there was not a significant increase in postoperative AKI of patients with low PaO2 (group 3 and 4) during the early period after CTS. We hypothesized that it might be related to these adapt changes. The animal study suggested that hypoxic preconditioning of animals in vivo increased hypoxic tolerance (32). Alexander Zarbock et al. found that among high-risk patients undergoing cardiac surgery, remote ischemic preconditioning compared with no ischemic preconditioning significantly reduced the rate of AKI and use of renal replacement therapy (33).

The limitation of this study includes the following aspects. First, the relatively small sample may have lacked the power to detect significant interactions. Second, Hemodynamic indexes which the study collected cannot directly represent renal perfusion. Third, the evaluation criteria of microcirculation and oxygen metabolism are still controversial at present, and the indexes we adopted in this paper need to be further verified.

\section{Conclusions}

In conclusion, low preoperative $\mathrm{PaO} 2$ did not significantly increase the incidence of $\mathrm{AKI}$, but patients with low $\mathrm{PaO} 2$ were more likely to have severe $\mathrm{AKI}$ and persistent $\mathrm{AKI}$. $\mathrm{Pv}$-aCO2/Ca-vO2 and $\mathrm{Pv}$-aCO2 are independent risk factors for postoperative $\mathrm{AKI}$ and $\mathrm{PV}-\mathrm{aCO} 2 / \mathrm{Ca}-\mathrm{vO} 2$ is an independent risk factor for persistent AKI. Monitoring $\mathrm{Pv}$-aCO2/Ca-v02 may offer some help for guiding AKI treatment.

\section{Abbreviations}

AKI, Acute Kidney Injury

CTS, Cardiothoracic Surgery

TOF, Tetralogy of Fallot

SCr, Serum Creatinine

KDIGO, Kidney Disease: Improving Global Outcomes

CVC, Central Venous Catheter

PICU, Pediatric Intensive Care Unit

RD, Renal Dysfunction

eGFR, Estimated Glomerular Filtration Rate

CPB, Cardiopulmonary Bypass

PaO2, Arterial Partial Pressure of Oxygen

Page 13/20 
Pa-v02, Central Arterial-to-Venous Oxygen Pressure Difference

O2 ER, Oxygen Extraction Ratio

Hct, Hematocrit

$\mathrm{Hb}$, Hemoglobin

PAP, Pulmonary Arterial Pressure

LVEF, Left Ventricular Ejection Fraction

LVFS, Fractional Shortening

ASA, American Society of Anesthesiologists grade

HR, Heart Rate

MAP, Mean Arterial Pressure

CVP, Central Venous Pressure

SaO2, Arterial Oxygen Saturation

Sa-v02, Central Arterial-to-Venous Oxygen Saturation Difference

Pv-aC02, Central Venous-to-Arterial Carbon Dioxide Difference

Ca-v02, Arterial-to-Central Venous 02 Content Difference

VIS, Vasoactive Inotrope Score

CHD, Congenital Heart Disease

A-CHD, Acyanosis Congenital Heart Disease

$\mathrm{C}-\mathrm{CHD}$, Cyanosis Congenital Heart Disease

$\mathrm{CN}$, Cyanotic Nephropathy

$\mathrm{Cl}$, Confidence Interval

$\mathrm{N}$, Number

\section{Declarations}

\section{Ethics approval and consent to participate}

Written informed consent was obtained from all participants, where participants are children (under 16 years old) from their parent or guardian. This permits the researchers to engage in research, the use of identifiable 
biospecimens and identifiable data during the peri-operative period and future follow-up without the requirement to obtain additional consent for the future storage, maintenance, or research uses, so long as the future activities are within the scope of the broad consent. The study protocols as well as the application form were fully reviewed, and we certify that this study did not raise any issues of patient risk or cause any harm to patients. We also certify that the study was strictly in accordance with the Declaration of Helsinki and International Ethical Guidelines for Health-related Research Involving Humans. This study was approved by the Ethics Committee of Xin Hua Hospital Affiliated to Shanghai Jiao Tong University School of Medicine (Approval No. XHEC-D-2020016).

\section{Consent for publication}

Written informed consent for publication was obtained from all participants

\section{Availability of data and materials}

The data that support the findings of this study are available from the corresponding author upon reasonable request.

\section{Competing interests}

The authors declare that they have no conflicts of interest.

\section{Funding}

This research did not receive any specific grant from funding agencies in the public, commercial, or not-for-profit sectors.

\section{Authors' Contributions}

YX contributed towards the study design, data collection and writing of the manuscript; YZ contributed towards the study design and literature search; ZL contributed towards the data collection and statistical analyses; LX contributed towards the statistical analyses; LC contributed towards data interpretation; WX contributed towards data interpretation and $X Z$ contributed towards data interpretation and writing of the manuscript. All authors approved the final version of the manuscript.

\section{Acknowledgements}

None.

\section{References}

1. O'Neal JB, Shaw AD, Billings FT. Acute kidney injury following cardiac surgery: current understanding and future directions. Crit Care. 2016;20(1):187. doi:10.1186/s13054-016-1352-z.

2. Thiele RH, Isbell JM, Rosner MH. AKI associated with cardiac surgery. Clin J Am Soc Nephrol. 2015;10(3):500-14. doi:10.2215/CJN.07830814.

3. Kataria Y. Epidemiology of acute kidney injury in critically ill children and young adults. Ann Clin Biochem. 2017;54(3):416. doi:10.1177/0004563217693559. 
4. Bellomo R, Kellum JA, Ronco C. Acute kidney injury. Lancet. 2012;380(9843):756-66. doi:10.1016/S01406736(11)61454-2.

5. James MT, Bhatt M, Pannu N, Tonelli M. Long-term outcomes of acute kidney injury and strategies for improved care. Nat Rev Nephrol. 2020;16(4):193-205. doi:10.1038/s41581-019-0247-z.

6. Amini S, Abbaspour H, Morovatdar N, et al. Risk Factors and Outcome of Acute Kidney Injury after Congenital Heart Surgery: A Prospective Observational Study. Indian J Crit Care Med. 2017;21(12):847-51. doi:10.4103/ijccm.IJCCM_459_16.

7. Terano C, Ishikura K, Miura M, et al. Incidence of and risk factors for severe acute kidney injury in children with heart failure treated with renin-angiotensin system inhibitors. Eur J Pediatr. 2016;175(5):631-7. doi:10.1007/s00431-015-2680-8.

8. Wu Q, Wang T, Chen S, et al. Cardiac protective effects of remote ischaemic preconditioning in children undergoing tetralogy of fallot repair surgery: a randomized controlled trial. Eur Heart J. 2018;39(12):102837. doi:10.1093/eurheartj/ehx030.

9. Liu H, Zheng SQ, Qian SC, et al. Haematocrit differences modify the association of cardiopulmonary bypass reoxygenation with acute kidney injury after paediatric Tetralogy of Fallot repair. Perfusion. 2020;35(4):2849. doi:10.1177/0267659119871777.

10. Asada D, Itoi T, Nakamura A, Hamaoka K. Tolerance to ischemia reperfusion injury in a congenital heart disease model. Pediatr Int. 2016;58(12):1266-73. doi:10.1111/ped.13022.

11. Lee JW, Ko J, Ju C, Eltzschig HK. Hypoxia signaling in human diseases and therapeutic targets. Exp Mol Med. 2019;51(6):1-13. doi:10.1038/s12276-019-0235-1. Published 2019 Jun 20.

12. Apitz C, Webb GD, Redington AN. Tetralogy of Fallot. Lancet. 2009;374(9699):1462-71. doi:10.1016/S01406736(09)60657-7.

13. Forman J, Beech R, Slugantz L, Donnellan A. A Review of Tetralogy of Fallot and Postoperative Management. Crit Care Nurs Clin North Am. 2019;31(3):315-28. doi:10.1016/j.cnc.2019.05.003.

14. Pan BL, Loke SS. Chronic kidney disease associated with decreased bone mineral density, uric acid and metabolic syndrome. PLoS ONE. 2018;13(1):e0190985. doi:10.1371/journal.pone.0190985.

15. Kellum JA, Lameire N, Aspelin P, et al. Kidney disease: Improving global outcomes (KDIGO) acute kidney injury work group. KDIGO clinical practice guideline for acute kidney injury. Kidney International Supplements, 2(1), 1-138. https://doi.org/10.1038/kisup.2012.1.

16. Xuming Mo Y, Liu Q, Xing, et al. Handbook of pediatric cardiac surgical intensive care [M]. Beijing: Science Press; 2009.

17. Koponen T, Karttunen J, Musialowicz T, Pietiläinen L, Uusaro A, Lahtinen P. Vasoactive-inotropic score and the prediction of morbidity and mortality after cardiac surgery. Br J Anaesth. 2019;122(4):428-36. doi:10.1016/j.bja.2018.12.019.

18. Rhodes LA, Erwin WC, Borasino S, Cleveland DC, Alten JA. Central Venous to Arterial CO2 Difference After Cardiac Surgery in Infants and Neonates. Pediatr Crit Care Med. 2017;18(3):228-33. doi:10.1097/PCC.0000000000001085.

19. Patel R, Solanki A, Patel H, Patel J, Pandya H, Sharma J. Monitoring Microcirculatory Blood Flow during Cardiopulmonary Bypass in Paediatric Cardiac Surgery Patients as a Predictor for Anaerobic Metabolism. J Clin Diagn Res. 2017;11(4):UC22-5. doi:10.7860/JCDR/2017/24360.9721. 
20. Mesquida J, Saludes P, Gruartmoner G, et al. Central venous-to-arterial carbon dioxide difference combined with arterial-to-venous oxygen content difference is associated with lactate evolution in the hemodynamic resuscitation process in early septic shock. Crit Care. 2015;19(1):126. Published 2015 Mar 28. doi:10.1186/s13054-015-0858-0.

21. Holt T, Filler G. Is it time for a multi-specialty approach to cardio-renal dysfunction in children with cyanotic congenital heart disease? Pediatr Nephrol. 2018;33(3):359-60. doi:10.1007/s00467-017-3805-2\.

22. Inatomi J, Matsuoka K, Fujimaru R, et al. Mechanisms of development and progression of cyanotic nephropathy. Pediatr Nephrol. 2006;21(10):1440-5. doi:10.1007/s00467-006-0220-5.

23. Greenwood SA, Mangahis E, Castle EM, et al. Arterial stiffness is a predictor for acute kidney injury following coronary artery bypass graft surgery. J Cardiothorac Surg. 2019;14(1):51. doi:10.1186/s13019-019-0873-3. Published 2019 Mar 7.

24. Park SK, Hur M, Kim E, et al. Risk Factors for Acute Kidney Injury after Congenital Cardiac Surgery in Infants and Children: A Retrospective Observational Study. PLoS One. 2016;11(11):e0166328. doi:10.1371/journal.pone.0166328. Published 2016 Nov 10.

25. Bai Y, Zhang Y, Yang S, et al. Protective effect of vascular endothelial growth factor against cardiopulmonary bypass-associated acute kidney injury in beagles. Exp Ther Med. 2018;15(1):963-9. doi:10.3892/etm.2017.5460.

26. Li J, Zhang G, Holtby HM, et al. Inclusion of oxygen consumption improves the accuracy of arterial and venous oxygen saturation interpretation after the Norwood procedure. J Thorac Cardiovasc Surg. 2006;131(5):1099-107. doi:10.1016/j.jtcvs.2005.10.057.

27. Ospina-Tascón GA, Madriñán HJ. Combination of 02 and C02-derived variables to detect tissue hypoxia in the critically ill patient. J Thorac Dis. 2019;11(Suppl 11):1544-50. doi:10.21037/jtd.2019.03.52.

28. Wan XY, Wei LL, Jiang Y, et al. Effects of time delay and body temperature on measurements of central venous oxygen saturation, venous-arterial blood carbon dioxide partial pressures difference, venous-arterial blood carbon dioxide partial pressures difference/arterial-venous oxygen difference ratio and lactate. BMC Anesthesiol. 2018;18(1):187. doi:10.1186/s12871-018-0655-9.

29. Shaban M, Salahuddin N, Kolko MR, et al. The Predictive Ability of PV-ACO2 Gap and PV-ACO2/CA-VO2 Ratio in Shock: A Prospective, Cohort Study. Shock. 2017;47(4):395-401.

doi:10.1097/SHK.0000000000000765.

30. Mukai A, Suehiro K, Kimura A, et al. Comparison of the venous-arterial CO2 to arterial-venous 02 content difference ratio with the venous-arterial $\mathrm{CO} 2$ gradient for the predictability of adverse outcomes after cardiac surgery. J Clin Monit Comput. 2020;34(1):41-53. doi:10.1007/s10877-019-00286-z.

31. Onk OA, Onk D, Ozcelik F, et al. Risk Factors for Acute Kidney Injury after Coronary Artery Bypass Surgery and Its Detection Using Neutrophil Gelatinase-Associated Lipocalin. Cardiorenal Med. 2016;6(3):216-29. doi:10.1159/000444099.

32. Lu GW, Cui XY, Zhao BM. Alteration of oxygen consumption and energy metabolism during repetitive exposure of mice to hypoxia. Neurochem Res. 1999;24(5):625-8. doi:10.1023/a:1021092023253.

33. Zarbock A, Schmidt C, Van Aken $\mathrm{H}$, et al. Effect of remote ischemic preconditioning on kidney injury among high-risk patients undergoing cardiac surgery: a randomized clinical trial. JAMA. 2015;313(21):2133-41. doi:10.1001/jama.2015.4189. 


\section{Figures}

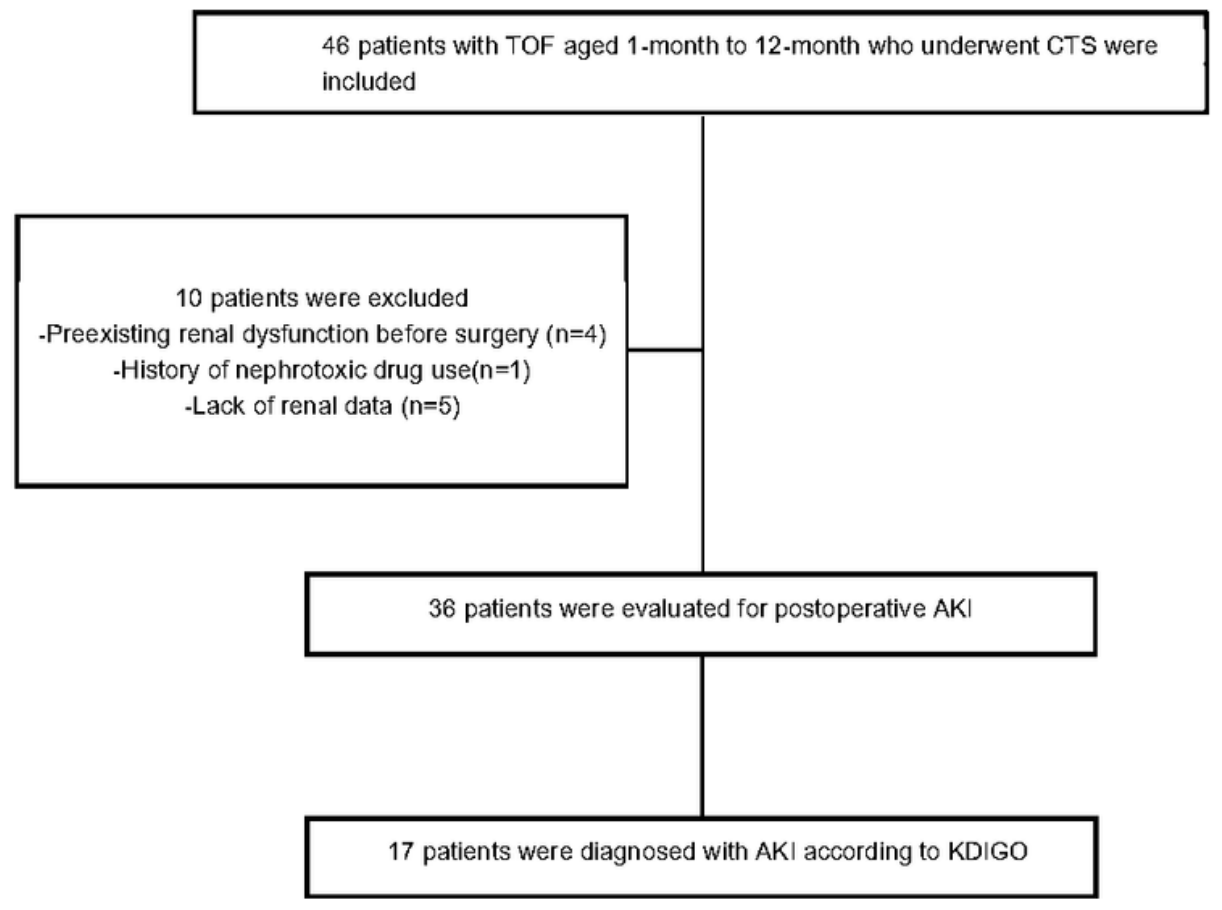

Figure 1

Flow diagram of the study participants TOF= Tetralogy of Fallot; CTS=cardiothoracic surgery; AKI=acute kidney injury; KDIGO=Kidney Disease: Improving Global Outcomes 


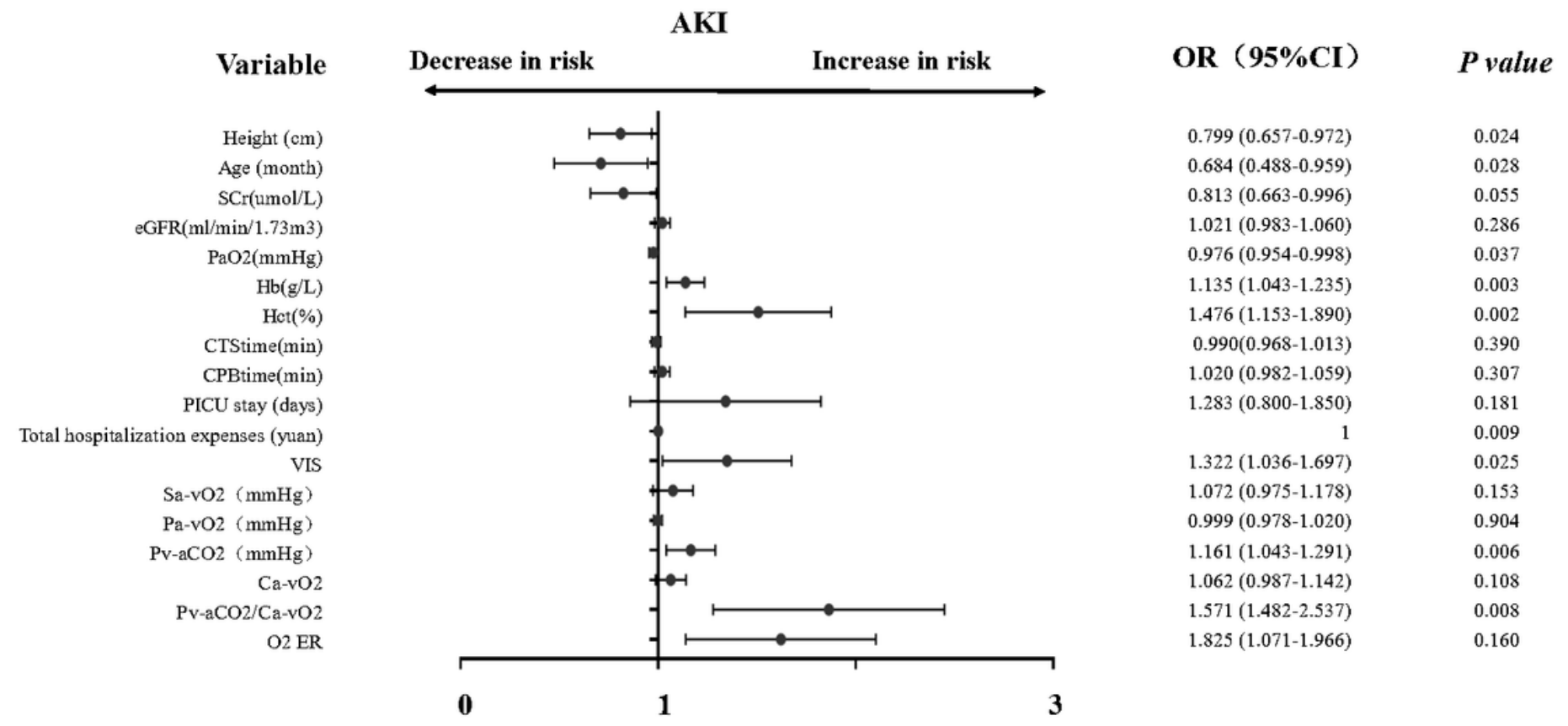

Figure 2

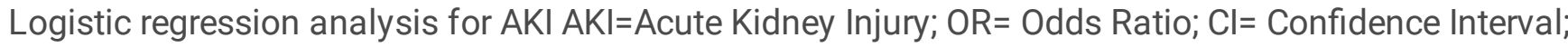
$\mathrm{PaO} 2=$ Arterial Partial Pressure of Oxygen; CTS = Cardiothoracic Surgery; CPB=Cardiopulmonary Bypass; SCr=Serum Creatinine; eGFR=estimated Glomerular Filtration Rate; PICU=Pediatric Intensive Care Unit; $\mathrm{Hct}=$ Hematocrit; $\mathrm{Hb}=$ Hemoglobin; VIS= Vasoactive Inotrope Score; SaO2= Arterial Oxygen Saturation; PVaCO2/Ca-vO2= the ratio of central venous-to-arterial carbon dioxide difference (Pv-aCO2) to arterial-to-central venous $\mathrm{O} 2$ content difference $(\mathrm{Ca}-\mathrm{vO} 2)$; O2ER=0xygen Extraction Ratio 


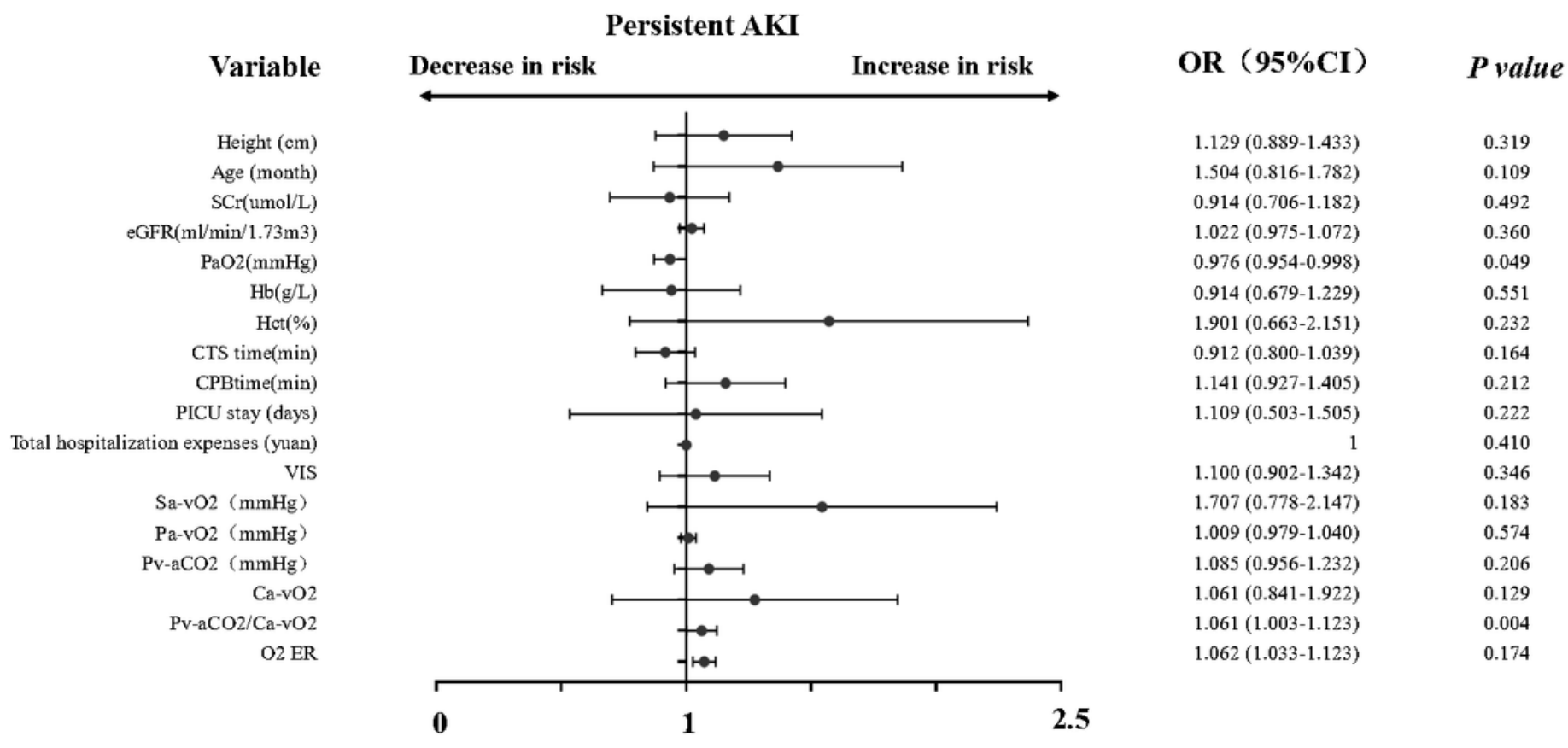

Figure 3

Logistic regression analysis for persistent $\mathrm{AKI} A \mathrm{AKI}=$ Acute Kidney Injury; OR= Odds Ratio; $\mathrm{Cl}=\mathrm{Confidence} \mathrm{Interval;}$ $\mathrm{PaO} 2=$ Arterial Partial Pressure of Oxygen; CTS = Cardiothoracic Surgery; CPB=Cardiopulmonary Bypass; SCr=Serum Creatinine; eGFR=estimated Glomerular Filtration Rate; PICU=Pediatric Intensive Care Unit; Hct=Hematocrit; $\mathrm{Hb}=$ Hemoglobin; VIS= Vasoactive Inotrope Score; SaO2= Arterial Oxygen Saturation; PvaCO2/Ca-vO2= the ratio of central venous-to-arterial carbon dioxide difference ( $\mathrm{Pv}$-aCO2) to arterial-to-central venous $\mathrm{O} 2$ content difference (Ca-v02) ; 02ER=0xygen Extraction Ratio.

\section{Supplementary Files}

This is a list of supplementary files associated with this preprint. Click to download.

- STROBEchecklist.docx 